\title{
WIDTH OF THE LATITUDE OF ACCEPTANCE AS A DETERMINANT OF ATTITUDE CHANGE ${ }^{1}$
}

\author{
ALICE H. EAGLY ${ }^{2}$ AND KATHLEEN TELAAK ${ }^{3}$
}

University of Massachusetts

\begin{abstract}
In a study examining the implications of social judgment theory for predicting individual differences in attitude change, a subject received a message advocating a position on birth control either mildly, moderately, or strongly discrepant from his own. For all three levels of discrepancy, the subjects with wide latitudes of acceptance on the issue showed greater attitude change than did subjects with either narrow or medium latitudes of acceptance. Since a mildly discrepant message was in the lattitude of acceptance even for narrow latitude subjects and a strongly discrepant message in the latitude of rejection even for wide latitude subjects, this finding cannot be reconciled with social judgment theory. Since correlations between latitude widths on birth control and two irrelevant issues were low and latitude width on the irrelevant issues did not relate to attitude change on birth control, width of the latitude of acceptance was interpreted as an issue-specific index of influenceability. Category width, though independent of latitude of acceptance width, also predicted attitude change: narrow categorizers changed their attitudes more than did either medium or broad categorizers.
\end{abstract}

Central to the social judgment approach to attitude change (Sherif, Sherif, \& Nebergall, 1965; Sherif \& Hovland, 1961) is the assumption that the structure of an individual's attitude is a determinant of receptivity to a persuasive communication. Social judgment theory specifies attitude structure by dividing the attitude dimension into "latitudes" or regions: a latitude of acceptance, which is the range of positions that an individual accepts, and a latitude of rejection, which is the range of positions that he rejects. ${ }^{4}$ According to the major hypothesis of

1 This study was partially supported by a faculty research grant to the first author from the Graduate School of the University of Massachusetts. The authors would like to thank Samuel Himmelfarb and Alan Wicker for their helpful comments on an earlier draft of this paper.

${ }^{2}$ Requests for reprints should be sent to Alice $H$. Eagly, Department of Psychology, University of Massachusetts, Amherst, Massachusetts 01002.

3 Now at Windham College.

4 Though, according to the more recent formulation of social judgment theory (Sherif et al., 1965), a latitude of noncommitment, which is the range of positions to which an individual is indifferent, frequently intervenes between the latitudes of acceptance and rejection, this third region occurred rarely among subjects in the present study (e.g., in the first session, only 6 of the 124 subjects were uncommitted on any of the birth control items). Thus, only the earlier two-region statement of the theory is considered in this paper. the theory, if a persuasive message is judged to be within the latitude of acceptance, attitude change toward the message occurs, while if a message is judged to be within the latitude of rejection, little or no attitude change occurs, or attitude change away from the message may even occur. Some general support for this hypothesis exists in studies finding that attitude change is maximal for messages either judged to be within the latitude of acceptance (Atkins, Deaux, \& Bieri, 1967) or located at the beginning of the latitude of rejection (Peterson \& Koulack, 1969). However, the implications of the theory for individual differences in susceptibility to social influence have received little attention. This study examines the proposition that individual differences in the relative widths of the latitudes of acceptance and rejection are a determinant of attitude change.

The assumption that location of a message in relationship to the latitudes determines attitude change suggests that under certain conditions persons with relatively wide latitudes of acceptance and narrow latitudes of rejection are more likely to change toward a message than persons with narrow latitudes of acceptance and wide latitudes of rejection. This effect would occur for a message moderately discrepant from subjects' initial posi- 
tions since it would tend to fall in the latitude of acceptance of wide latitude of acceptance subjects but in the latitude of rejection of narrow latitude of acceptance subjects. According to the theory, neither a slightly discrepant message, since it is likely to be in the latitude of acceptance for all subjects, nor an extremely discrepant message, since it is likely to be the latitude of rejection for all subjects, can generate differences in response.

Two studies point to the possible significance of individual differences in latitude width. Zimbardo (1960) reported, as a subsidiary finding of a study on involvement, that subjects who changed their opinions toward a friend's position had initially accepted a wider range of statements on the issue and rejected a narrower range than had subjects who did not change toward the friend. Yet this finding is ambiguous since the subjects in one experimental condition received a message tailored to be in the latitude of acceptance, while the subjects in another condition received a message tailored to be in the latitude of rejection-a procedure which probably caused the messages presented to the wide latitude of acceptance subjects to be more discrepant from subjects' initial positions than were the messages presented to the narrow latitude of acceptance subjects. Nor was it clear in the Zimbardo study whether latitude width was independent of extremity of initial position. In an unpublished study by Miller and Devine (reported in Kiesler, Collins, \& Miller, 1969), subjects with typically broad latitudes of rejection (assessed across 30 attitude dimensions) resisted persuasion more strongly than did subjects with typically narrow latitudes of rejection. Though these two studies are suggestive, they do not provide definitive evidence that latitude width affects attitude change, nor is there sufficient knowledge concerning the theoretically crucial issue of the generality of the effect of latitude width across levels of discrepancy.

An additional issue addressed by this study is whether latitude width is a function of more general attitudinal or cognitive dispositions. One aspect of this issue is whether one's distribution of attitude positions between the latitudes of acceptance and rejection is a stable general tendency of the person rather than issue specific. To the extent that latitude width is a general rather than an issue-specific characteristic, latitude widths would tend to be positively correlated across social issues, and a general measure of latitude width, such as that used by Miller and Devine, would relate to attitude change.

We can also go beyond the attitude literature to examine research on cognition and readily find several representations of the idea that individuals differ in the range of events that they are willing to consider acceptable exemplars of a category. For example, Pettigrew (1958) formulated the Category Width scale, which assesses, across a number of concepts, the extent to which an individual places divergent events within a single category. In addition, there are measures of "cognitive style" conceptualized along somewhat similar lines. For example, leveling-sharpening (Holtzman, 1953; Holtzman \& Klein, 1954) is a potentially relevant style since sharpeners are assumed to maximize stimulus differences and to be attuned to small gradients of differences between stimuli, while levelers minimize stimulus differences and do not attend to small differences between stimuli. In a related cognitive style tradition, Gardner (1953) examined individual differences in "equivalence range." Two representatives of the cognitive tradition were included in the present study in order to evaluate their relationship to the concepts of latitudes of acceptance and rejection.

\section{METHoD}

\section{Overview}

In the first session, a questionnaire determined a subject's initial attitudes and latitudes of acceptance and rejection on three social issues. In a second session, a subject completed several measures of cognitive structure, then received a persuasive communication either slightly, moderately, or strongly discrepant from his initial attitude on one of the social issues, and, finally, responded to a questionnaire that assessed attitude change and several other responses to the communication.

\section{Subjects}

The subjects participating in both sessions were 124 University of Massachusetts students (65 males 
and 59 females) enrolled in an introductory psychology course that required participation in experiments.

\section{Procedure}

In the first session, held prior to an optional introductory psychology movie, 550 students completed a questionnaire containing a Sherif-type attitude scale (described below) for each of three social issues-birth control, legalization of marijuana, and gun control. Since students were allowed to choose the experiments they would participate in, the questionnaire also asked the respondent to indicate whether he would be a subject in an experiment concerning communication processes and opinions on current social issues. Of the 178 persons who voluntered for the experiment, the 174 who were pro birth control constituted the population from which the subjects were obtained. Due to absences, scheduling conflicts, etc., 124 subjects were actually run (in groups of 4) in the second session.

At the second session, the experimenter informed the subjects that the earlier questionnaire enabled her to find a topic on which there was a wide diversity of opinions and that the purpose of the second session was to study communication between subjects, who would be divided into partners. Though a subject was told he would not know which of the others was his partner, the experimenter further lessened the probability that a subject might think that he was communicating with a friend by asking whether any of the subjects knew one another and informing anyone who did that he would not be communicating with his friend but with another subject. Then subjects completed a questionnaire containing several measures of cognitive structure described as measures of "ways or styles of thinking..." that had a possible effect on the communication process.

After the subjects were sent to separate rooms, the experimenter asked each one to write a statement $\frac{1}{2}-1$ page long for his partner to read in which he expressed his honest and frank feelings about birth control. The experimenter collected each finished statement, apparently in order to exchange it with a message written by another subject. Instead, she presented each subject with a handwritten prescaled communication (described below) randomly selected to be either slightly, moderately, or strongly discrepant from his initial attitude on birth control.

The experimenter instructed the subject to read his partner's statement carefully. After about $2 \mathrm{~min}$ utes, the experimenter collected the message and gave the subject a questionnaire that she described as "designed to assess your reactions to the communication process." To justify the inclusion of attitude scales on the questionnaire, the experimenter mentioned that she thought that communication might be affected by one's stand on social issues and explained that some of the scales were similar to those filled out earlier but that it was necessary to measure "how you feel about social issues at precisely the same time you are involved in the com- munication process." The questionnaire contained a Sherif-type attitude scale for each of five social issues (the three from the first session plus two "filler" issues) and measures of judgment of the message, evaluation of the message and its source, and interpretation of the purpose of the experiment.

\section{Persuasive Messages}

A large pool of pro- and anti-birth-control messages had been prepared to make it possible to create as nearly as possible, a 1.0-point discrepancy from each subject's most preferred position in the slight condition, a 3.5-point discrepancy in the moderate condition, and a 7.5-point discrepancy in the strong condition. These discrepancy values were chosen so that a slightly discrepant message would generally be in a subject's latitude of acceptance, an extremely discrepant message in the latitude of rejection, and a moderately discrepant message, near the border between the latitudes of acceptance and rejection.

Each message in the pool of messages had been judged on an 11-point scale of favorability to birth control by 35 pretest subjects who were paid volunteers from introductory psychology courses. Scale values were derived according to Thurstone's equalappearing intervals technique. (Sample persuasive messages appear in Table 1.) All messages used in the second session were more opposed to birth control than were the subjects' initial attitudes.

\section{Measurement of Cognitive Structure}

Latitudes of acceptance and rejection. Three nine-item Sherif-type attitude scales (e.g., Sherif \& Hovland, 1961) were constructed, one each for birth control, legalization of marijuana, and gun control. Scale values for the items had been derived by an equal-appearing intervals procedure from judgments of a larger set of items by the same 35 pretest subjects who judged the messages. Nine items with scale values spaced approximately evenly along the scale were chosen for each of the scales and ordered from pro to anti. (Sample items for the birth control scale appear in Table 1.) In both the first and second sessions, a subject selected the statement that came closest to his own stand, the other statements that he agreed with, the most objectionable statement, and the other statements that he disagreed with.

Though Sherif defined latitude width in terms of the number of statements accepted and rejected, a more exact definition-the scalar distance between items in a latitude-was employed. Accordingly, width of the latitude of acceptance was the scalar distance between the most pro and the most anti statement that a subject agreed with (or chose as the statement closest to his own stand). Width of the latitude of rejection was taken as the complement of width of the latitude of acceptance since it encompassed the statements not in the lattitude of acceptance. 
TABLE 1

Sample Persuasive Messages and Sample Items for Asseissing the Subject's Own Position and Latitude Width

\begin{tabular}{l|l} 
An individual's sexual activity is his or her own business. The definition of morality is defined by young \\
people today in such different ways than that of their parents, and their behavior would be no different with \\
or without birth control products. Because this decision is such a personal thing and cannot be dictated by \\
others, birth control products, including the pill, should be made available to people of reasonable age. In \\
spite of the fact that our liberal society should protect the individual's rights to make such crucial decisions \\
for himself, we cannot and should not condone birth control for youngsters. Therefore the question of birth \\
control should be an individual decision, with such products available to young adults of reasonable age \\
because their sexual behavior would be no different with or without artificial birth control. \\
Birth control is unnatural and should not be practiced. If we were meant to practice birth control we \\
would have a built-in system to take care of such things. The world has done quite all right up to now. \\
When you stop to think how beautiful nature is you realize birth control has no place in it. Man and woman \\
love each other and in the process of expressing this love they create another human being who is a com- \\
bination of both of them. The man protects the woman and the woman protects the unborn child. Nature \\
has the whole thing arranged so beautifully and birth control has no place in it. Birth control in any way is \\
unnatural and should not be practiced. If man wants to live a rich and full and beautiful life as nature \\
intended, he should not use such artificial devices.
\end{tabular}

Sample items

Birth control devices should be available to everyone, and the government and public welfare agencies should encourage the use of such devices.

To curtail indiscriminate premarital sex, birth control products should be distributed by doctors to married people only.

All birth control devices should be illegal and the government should prevent them from being manufactured.

a On an 11-point scale on which 1 is the favorable and 11 the unfavorable pole.

Other structural mesasures. Category width was assessed by the 10 items from Pettigrew's (1958) Category Width scale that he reported had the strongest relationships to criterion laboratory tasks. The leveling-sharpening tendency was assessed by the 16-item Assimilating-Differentiating scale devised by Child and revised by Baron (1965), which focuses on self-reported differences in memory organization. These items were embedded among irrelevant items.

\section{Measures of Dependent Variables}

Attitude change. Attitude change on an issue was defined as the difference in scale value between the item checked as closest to one's own stand in the first session and the item checked as closest in the second session. Change in latitude of acceptance width or in the average scale value of items in the latitude of acceptance was less relevant because selection of subjects on latitude width would introduce a regression effect for variables initially correlated with latitude width.

Message judgment. In judging the position taken by the message he received, a subject responded on the same 11-point scale used by the pretest subjects who judged the messages and scale items. A subject's judgment of his message was compared to its scale value to determine whether he had assimilated it (perceived it as closer to his own position) or contrasted it (perceived it as further from his own position).

Evaluation of the message and source. On 7-point rating scales, a subject indicated how effective, well written, and fair the communication was and how unbiased, well informed, and expressive his communication partner was.

Suspicion. An open-ended question asking subjects to give their interpretation of the purpose of the experiment was administered after the other measures. Answers were coded (independently by two raters) to determine if any doubted the authenticity

5 It might be noted that assimilation and contrast are defined in relationship to the standard provided by judges, who themselves could have assimilated or contrasted the messages in relationship to some "objective" standard. However, any effect of latitude of acceptance width on judgment would still be interpretable, though, strictly, only as a difference in the placement of messages. Ideally, interpretation in terms of assimilation and contrast would be based on an unambiguous definition of the zero-point (veridicality) on the assimilation-contrast scale. 
of the message. The six subjects who indicated some awareness that the message might not have been written by their partner were not retained in the analysis: two received a slightly discrepant, one a moderately discrepant, and three a strongly discrepant message.

\section{RESULTS}

\section{Latitude Width and the Placement of Messages}

The distribution of width of latitude of acceptance scores for the birth control issue was divided into narrow, medium, and wide classes so as to equalize as nearly as possible the number of subjects in each latitude width category. However, the thirds were necessarily unequal because 72 of the 118 subjects chose a single most popular "medium" width (though these subjects differed among themselves in which position they most preferred).

The distance between a subject's initial most preferred position and the beginning of his latitude of rejection was calculated separately for the narrow, medium, and wide latitude subjects: This distance was, on the average, 2.26 points for the narrow subjects, 3.68 points for the medium subjects, and 5.07 for the wide subjects. The slightly discrepant messages, which had a mean discrepancy of .96 , had scale values that placed them within the latitude of acceptance for all subjects. The strongly discrepant messages, which had a mean discrepancy of 7.34 , had scale values that placed them within the latitude of rejection for all subjects with the exception of two subjects, for whom the message fell only slightly beyond the boundary of the latitude of rejection. As expected, the moderately discrepant messages, which had a mean discrepancy of 3.74 , had a more variable loca-

TABLE 2

Mean Attitude Change as a Function of Latitude of Acceptance Width and Discrepancy Size

\begin{tabular}{|c|c|c|c|c|}
\hline \multirow{2}{*}{$\begin{array}{l}\text { Latitude of } \\
\text { acceptance } \\
\text { width }\end{array}$} & \multicolumn{4}{|c|}{ Discrepancy } \\
\hline & Slight & Moderate & Strong & $M$ \\
\hline $\begin{array}{l}\text { Narrow } \\
\text { Medium } \\
\text { Wide } \\
\qquad M\end{array}$ & $\begin{array}{r}-.07(8) \\
.24(27) \\
.68(5) \\
.24(40)\end{array}$ & $\begin{array}{r}.18(9) \\
-.09(24) \\
1.25(8) \\
.23(41)\end{array}$ & $\begin{array}{r}-.49(8) \\
.10(21) \\
.42(8) \\
.04(37)\end{array}$ & $\begin{array}{r}-.11(25) \\
.09(72) \\
.80(21)\end{array}$ \\
\hline
\end{tabular}

* Cell $n s$ are given in parentheses, tion in relationship to the latitudes: For $100 \%$ of the narrow latitude subjects, the message was in the latitude of rejection; for $42 \%$ of the medium latitude subjects, the message was between the last statement in the latitude of acceptance and the first statement in the latitude of rejection, and for $58 \%$ it was in the latitude of rejection; for $38 \%$ of the wide latitude subjects it was in the latitude of acceptance; for $38 \%$ it was between the latitudes of acceptance and rejection; and for $25 \%$ it was in the latitude of rejection.

\section{Relationships of Latitude of Acceptance Width to Responses}

The correlation between the width of the latitude of acceptance and the initial most preferred position on the birth control issue did not approach significance (nor did the correlation between any other structural measure and initial position). Consequently, attitude change was treated in all analyses as a simple difference between initial and final most preferred positions.

Mean attitude change scores appear in Table 2, where positive numbers indicate change toward the message and negative numbers change away from the message. Analysis of variance (by the unweightedmeans method of treating unequal $n \mathrm{~s}$ ) indicated that only the main effect of latitude width was significant $(F=8.47, d f=2 / 109$, $p<.001$ ). Tukey $(b)$ (Winer, 1962) tests of the differences between the three levels of latitude width revealed that the wide latitude subjects changed significantly more than both the medium and the narrow latitude subjects $(p<.01)$, but narrow and medium subjects did not differ from each other. Change differed significantly from zero only in the wide latitude condition $(t=2.16, d f=20$, $p<.05)$. Neither the main effect of discrepancy size $(F=1.84)$ nor the interaction between latitude width and discrepancy size $(F=1.10)$ was significant, and the quadratic trend (Gaito, 1965) observed among the wide latitude subjects only approached significance $(F=2.88, d f=1 / 109, p<.10) .^{6}$

${ }^{\circ}$ Change in latitude of acceptance width was also examined as a function of discrepancy (though the 
To determine whether the attitude change findings might be test-retest phenomena unrelated to the reception of a message, attitude change on each of the irrelevant issues (gun control and legalization of marijuana) was examined as a function of latitude of acceptance width on the birth control issue, but no signficant relationships were obtained. Also, a measure of latitude width formed by summing widths over the two irrelevant issues failed to relate to attitude change on birth control.

When the relationship of birth control latitude of acceptance width to the other dependent variables (message judgment, source evaluation, message evaluation) was examined, no significant main effects of latitude width or interactions of latitude width with discrepancy were obtained. However, the main effect of message discrepancy was significant for all three variables. The discrepancy effect in the message judgment data $(F=4.07, d f=2 / 109, p<.02)$ indicated that the subjects contrasted the message more as it became more discrepant (slight discrepancy, $\bar{X}=.10 ;$ moderate discrepancy, $\bar{X}=-.15 ;$ strong discrepancy, $\bar{X}=-1.28$, where positive numbers signify assimilation and negative numbers signify contrast). Only the linear trend was significant, and displacement of the message differed significantly from zero only in the strong discrepancy condition. In addition, discrepancy related significantly to message evaluation $(p<.02)$ and to source evaluation $(p<.001)$ with significant linear trends indicating that evaluation became more negative as discrepancy size increased.

Attitude change on the birth control issue was also examined as a function of judgment of the message: Each subject was classified according to whether he judged the message to be within his latitude of acceptance or

regression effect had prevented interpretation of this dependent variable as a function of latitude width). Overall, the pattern was fairly similar to that shown in Table 2, though the discrepancy main effect was significant $(p<.01)$. It was characterized in the narrow and wide latitude width conditions by significant inverted- $U$ quadratic trends. There was a slight linear decreasing function among the moderate width subjects. beyond it. Subjects who judged it within the latitude of acceptance changed more $(\bar{X}=.40)$ than those who judged it outside of the latitude of acceptance $(\bar{X}=.00$; $t=2.25, d f=11.7, p<.05$ ).

Analyses including sex as an independent variable demonstrated not only that the relationships of latitude width to the dependent variables did not differ between the sexes but also that the sexes did not respond differently to the messages (i.e., there were no main effects of sex).

\section{Relationships of Other Structural Measures to Responses}

The Assimilating-Differentiating scale showed only weak and nonsignificant relationships to response, and those weak patterns that did appear tended to differ between the sexes.

The category width distribution was divided as equally as possible into thirds, and the resulting data were treated by analysis of variance. Mean change was .52 for the narrow subjects $(n=39), .04$ for the medium subjects $(n=40)$, and -.03 for the wide subjects $(n=39)$. In the analysis of variance, the main effect of category width was significant $(F=4.62, d f=2 / 109, p<.02)$, while neither the main effect of message discrepancy $(F=1.00)$ nor the interaction $(F<1)$ approached significance. Tukey $(b)$ tests of the differences between the three levels of category width revealed that the narrow categorizers changed significantly more than both the wide and the medium categorizers $(p<.05)$, but the wide and medium categorizers did not differ from each other. Attitude change differed from zero only in the narrow condition $(t=2.62, d f=38$, $p<.02)$.

Category width failed to relate to message judgment and showed only weak relationships to source and message evaluation: Evaluation of the source $(p<.08)$ and of the message $(p<.13)$ became somewhat more positive as category width decreased. Also, analyses including sex as an independent variable showed that the effect of category width was general across the sexes. 


\section{Relationships between Structural Measures}

Only one of the three correlations between the measures of width of the latitudes of acceptance was significant (between the gun control and marijuana issues, $r=.22$, $d f=116, p<.05)$. Only one of the other correlations between structural measures was significant (between the Assimilating-Differentiating scale and width of the marijuana latitude of acceptance, $r=.27, d f=116$, $p<.01$ ).

\section{Discussron}

The principal finding of the present study is that persons with wide latitudes of acceptance on an issue changed their attitudes toward a persuasive communication on that issue, while persons with narrow or medium latitudes of acceptance did not change. This finding was true regardless of how discrepant the communication was from the subjects' initial positions.

\section{Implications for Social Judgment Theory}

The fact that the greater influenceability of wide latitude persons was general across the three levels of message discrepancy has negative implications for the social judgment model of the attitude change process. Since messages in the slight discrepancy condition were in the latitude of acceptance even of the narrow latitude subjects, and messages in the strong discrepancy condition were in the latitude of rejection even of the wide latitude subjects, latitude width should have made little difference in these two experimental conditions. Differences in attitude change would be expected to be stronger in the moderate discrepancy condition than in either of the other conditions since only in this condition did variation in latitude width make a difference in the latitude in which messages were located.

One might possibly attempt to rescue social judgment theory by a more complex hypothesis derived from the assumption that change reaches a maximum level near the acceptance/rejection border and that this maximum is corstant over levels of latitude width. Then one could hypothesize a steeper ascending and a flatter descending discrepancy function the narrower the latitude of acceptance. However, this hypothesis would be compatible in the slight discrepancy condition only with a negative relationship between latitude width and attitude change.

To examine further the implications of social judgment theory, the function of assimilation and contrast must be considered in relationship to the attitude change findings. It might first be noted, however, that the role of judgmental processes is not entirely clear-cut within the structure of social judgment theory. Assimilation and contrast, as well as attitude change, could be interpreted as responses to messages of differing actual discrepancies, when the position of the message is defined by some external criterion and predictions for differing discrepancies are made in relationship to each subject's latitudes of acceptance and rejection. Then attitude change and perceptual displacement of the message from its actual position can be considered alternate responses that covary as discrepancy increases because they are both a function of discrepancy size.

It is, however, more usual to interpret social judgment theory as treating assimilation and contrast as responses that mediate attitude change. Then the perceived location (which may or may not be veridical) of a message in relationship to the boundaries of the latitudes determines attitude change. The idea that assimilation and contrast mediate attitude change has been stated most clearly in relationship to social judgment theory by Kiesler, Collins, and Miller (1969), in their two-stage model of attitude change, and by Atkins, Deaux, and Bieri (1967). Some support for the two-stage notion exists in the present finding that messages judged to be in the latitude of acceptance were somewhat more persuasive than messages judged to be beyond its boundaries, while actual discrepancy, as manipulated experimentally, had no significant effect on attitude change. However, the relationship between message judgment and attitude change was not very strong, and it failed to account for the relationship of latitude width to attitude change since the subjects' judgments of the message were independent of latitude width. Thus, the 
mediational role of message judgment was not confirmed insofar as the effect of latitude width on attitude change was concerned. For this reason, it does not matter in this study whether actual or perceived location of the message is used to interpret the effect of latitude width on attitude change (except for the fact that the strongly discrepant messages were perceived to be even further into the latitude of rejection than they actually were, i.e., a significant contrast effect). ${ }^{7}$

Thus, there are two important ways in which the latitude width analysis raises serious questions about the extent to which social judgment theory accounts for the attitude change process: (a) the absence of an interaction between latitude width and discrepancy size in the attitude change data and (b) the fact that latitude width related only to attitude change and not to the other responses (message judgment, message evaluation) that the theory states are also a function of the location of the message in relationship to the latitudes. It appears that social judgment theory is in the unusual position of providing a structural variable-relative width of the latitudes of acceptance and rejection-that had a significant impact on attitude change yet formulating no mechanism that adequately explains the present data. Clearly, social judgment theory gives too literal an interpretation to the latitude construct in imputing significance to the latitudes' exact locations on the attitude dimension.

If one looks beyond the formal structure of social judgment theory to understand why latitude width did affect attitude change, one possible explanation is that latitude width assesses one's tolerance for the views of others in a particular area-his willingness to consider admirable and reasonable views

7 This contrast effect may have occurred because a strongly anti-birth-control message was more unexpected in the experimental situation, which employed students as communication sources, than it was in the judgment situation, in which subjects expected to receive the full range of viewpoints on the issue. See attribution theory's (Jones \& Davis, 1965) suggestion that an unexpected position is judged more extreme than the same position when it is expected. not synonymous with his own. This openness to other's views implied by indicating that many positions are acceptable and few unacceptable on a certain issue may create a vulnerability to the persuasive impact of messages advocating views discrepant from one's own on that issue. What is crucial is the higher level of influenceability for messages of all discrepancies rather than the location of a message in relationship to the boundaries of the latitudes of acceptance and rejection.

There are, of course, other possible interpretations consistent with the findings. For example, width of the latitude of acceptance may increase as one becomes more uncertain concerning what his position is, and this uncertainty may be responsible for the greater amount of attitude change. Similarly, latitude width may represent degree of confidence in one's initial opinion or degree of commitment to it or perhaps involvement in the issue. Then the relationship to attitude change might indicate that a more confident, committed, or involved subject is less willing to accommodate his views to those of another person with whom he is interacting.

The present study also examined the generality of latitude width and suggests that it is largely an issue-specific index of influenceability, Powell's (1966) finding that width of the latitude of rejection related to dogmatism implies that latitude width is a function of more general personality dimensions. ${ }^{8}$ However, correlations between latitude width on the three issues employed in the present study provided little evidence that latitude width is general across issues. Also, latitude width on the irrelevant issues failed to predict attitude change. Furthermore, the low correlations between the width of the latitudes of acceptance and each of the other two structural measures yielded little evidence that latitude width is a function of more genera? tendencies to operate as a narrow-band versus a wide-band cognizer. Thus, the present

8 However, the fact that dogmatism was also correlated with extremity of own position suggests that the correlation between dogmatism and latitude of rejection may be artifactual. See Markley's (1971) discussion of latitude of rejection as an artifact of own position. 
study suggests that, while latitude width does account for individual differences in attitude change, it is not the type of construct that one would ordinarily think of as a personality variable, that is, a relatively general disposition of the person.

\section{Category Width}

Category width's significant effect on attitude change indicated that narrow categorizers changed their attitudes toward the message while wide and medium categorizers did not. This finding is generally consistent with earlier work involving category width and social influence: Steiner and Johnson (1965) found narrow categorizers more likely than wide categorizers to use conformity as a response to influence, and Eagly (1969) reported that male subjects who were both narrow categorizers and intolerant of inconsistency changed their attitudes more strongly than did other males. Thus, the present study provides additional evidence for Eagly's (1969) view that a narrow categorizer has a greater need to resolve inconsistency than does a broad categorizer and therefore responds more strongly by whatever discrepancy-reducing responses are available-attitude change in the present study.

This interpretation of category width also agrees with Rosen's (1961) finding that narrow categorizers were more likely to employ a dissonance-reducing response, seeking information that supported a decision, than were wide categorizers. It should be noted that in the present study the category width effect occurred for both sexes, while the earlier investigations have employed either male subjects only (Steiner \& Johnson, 1965) or obtained a category width finding significant only among males (Eagly, 1969; Rosen, 1961).

Accordingly, latitude width and category width apparently function along rather different lines. While latitude of acceptance width is an issue-specific index of influenceability, category width has related to response to several issues across widely divergent experimental situations. Category width apparently assesses a very general disposition of the person-the strength of the tendency to resolve inconsistency by whatever responses are salient in a given situation. Thus, latitude of acceptance width and category width have a phenotypic similarity if one thinks of both of them as assessing the range of discriminably different events considered exemplars of a category. But their impact works in opposite directions (since narrow category width but a wide latitude of acceptance increase attitude change) and arises from different sources.

\section{Message Reception as a Mediator of Attitude Change}

Finally, some general comments are in order concerning the role of message reception processes in predicting attitude change, especially in view of McGuire's (1968) "reception mediator" model of the relationship between personality and influenceability. This model attempts to establish a general framework for predicting individual differences in influenceability by assuming that a personality variable accounts for individual differences in how well a subject comprehends a message as well as in how much he yields to what he understands. The fact that social judgment theory narrows comprehension to a single aspect, comprehension of the overall position taken by the message, suggests that message reception might well be separable into at least two aspects-judgment of the overall position of the message and comprehension of the arguments and facts supporting this position. The present findings yielded no evidence that the effect of latitude width or of category width on attitude change was mediated by judgmental displacements of the overall position of the message. Thus, this study highlights the need for research that clarifies the conditions under which these reception processes have the mediational role suggested by these theoretical frameworks.

\section{REFERENCES}

Atkins, A. L., Deaux, K. K., \& Bieri, J. Latitude of acceptance and attitude change: Empirical evidence for a reformulation. Journal of Personality and Social Psychology, 1967, 6, 47-54.

BARON, R. M. Cognitive basis of attitude change as a function of motivational, stylistic, and stimulus factors. Journal of Personality and Social Psychology, 1965, 2, 219-230. 
EAGLY, A. H. Responses to attitude-discrepant information as a function of intolerance of inconsistency and category width. Journal of Personality, 1969, 37, 601-617.

GaIto, J. Unequal intervals and unequal $n$ in trend analysis. Psychological Bulletin, 1965, 63, 125-127.

Gardner, R. W. Cognitive styles in categorizing behavior. Journal of Personality, 1953, 22, 214-233.

Holzman, P. S. The relation of assimilation tendencies in visual, auditory, and kinesthetic timeerror to cognitive attitudes of leveling and sharpening. Journal of Personality, 1953, 22, 375-394.

Holzman, P. S., \& KLeIN, G. S. Cognitive systemprinciples of leveling and sharpening: Individual differences in assimilation effects and in visual time-error. Journal of Psychology, 1954, 37, 105122.

Jones, E. E., \& Davis, K. E. From acts to dispositions. In L. Berkowitz (Ed.), Advances in experimental social psychology. Vol. 2. The attribution process in person perception. New York: Academic Press, 1965.

KIEsler, C. A., Collins, B. E., \& Miller, N. Attitude change: $A$ critical analysis of theoretical approaches. New York: Wiley, 1969.

MarRley, O. W. Latitude of rejection: An artifact of own position. Psychological Bulletin, 1971, 75, $357-359$.

MCGurre, W. J. Personality and susceptibility to social influence. In E. F. Borgatta \& W. W. Lambert (Eds.), Handbook of personality theory and research. Chicago: Rand McNally, 1968.
Peterson, P. D., \& Kovlack, D. Attitude change as a function of latitudes of acceptance and rejection. Journal of Personality and Social Psychology, 1969, 11, 309-311.

Petcigrew, T. F. The measurement and correlates of category width as a cognitive variable. Journal of Personality, 1958, 26, 532-544.

Powel, F. A. Latitudes of acceptance and rejection and the belief-disbelief dimension: $A$ correlational comparison. Journal of Personality and Social Psychology, 1966, 4, 453-457.

Rosen, S. Postdecision affinity for incompatible information. Journal of Abnormal and Social Psychology, 1961, 63, 188-190.

SherIF, C. W., Sherif, M., \& Nebergall, R. E. Attitude and attitude change. Philadelphia: Saunders, 1965.

SHerIF, M., \& Hovtand, C. Social judgment. New Haven: Yale University Press, 1961.

Steiner, I. D., \& Johnson, H. H. Category width and responses to interpersonal disagreements. Journal of Personality and Social Psychology, 1965, 2, 290-292.

WINER, B. J. Statistical principles in experimental design. New York: McGraw-Hill, 1962.

ZnMbardo, P. G. Involvement and communication discrepancy and determinants of opinion conformity. Journal of Abnormal and Social Psychology, $1960,60,86-94$.

(Received June 1, 1971) 